Available on line at www.rac.es/racsam Geometry and Topology
REVISTA DE LA REAL ACADEMIA DE CIENCIAS EXACTAS, FISICAS Y NATURALES.

$\mathbb{S E R} \mathbb{R} \mathbb{A}: \mathbb{M A T E M A T I C A S}$

Madrid (España / Spain)

\title{
On gonality automorphisms of $p$-hyperelliptic Riemann surfaces
}

\author{
Ewa Tyszkowska
}

\begin{abstract}
A compact Riemann surface $X$ of genus $g>1$ is said to be a $p$-hyperelliptic if $X$ admits a conformal involution $\rho$ for which $X / \rho$ has genus $p$. This notion is the particular case of so called cyclic $(q, n)$-gonal surface which is defined as the one admitting a conformal automorphism $\delta$ of order $n$ such that $X / \delta$ has genus $q$. It is known that for $g>4 p+1, \rho$ is unique and so central in the automorphism group of $X$. We give necessary and sufficient conditions on $p$ and $g$ for the existence of a Riemann surface of genus $g$ admitting commuting $p$-hyperelliptic involution $\rho$ and $(q, n)$-gonal automorphism $\delta$ for some prime $n$ and we study its group of automorphisms and the number of fixed points of $\delta$. Furthermore, we deal with automorphism groups of Riemann surfaces admitting central automorphism with at most 8 fixed points. The condition on the small number of fixed points of such an automorphism is justified by the study of $p$-hyperelliptic surfaces.
\end{abstract}

\section{Sobre automorfismos de gonalidad de superficies de Riemann $p$-hiperelípticas}

Resumen. Una superficie de Riemann compacta $X$ de género $g>1$ se dice $p$-hiperelíptica si $X$ admite una involución conforme $\rho$, tal que $X / \rho$ tiene género $p$. Las superficies $p$-hiperelípticas son un caso particular de las superficies $(q, n)$-gonales cíclicas que se definen como aquellas superficies que admiten un automorfismo conforme $\delta$ de orden $q$ y de modo que $X / \delta$ tiene género $q$. En este trabajo nos restringiremos al caso en que $q$ es un número primo mayor que 2 . Es un hecho conocido que si $g>4 p+1$, la involución $\rho$ es única y central en el grupo de automorfismos de $X$. Obtenemos condiciones necesarias y suficientes sobre $p$ y $g$ para la existencia de superficies de Riemann de género $g$ que admiten una involución $p$-hiperelíptica y un automorfismo $(q, n)$-gonal que conmutan. Se determina la presentación de un cociente de los grupos de automorfismos de las superficies de Riemann que admiten un automorfismo $(q, n)$-gonal que sea central y con 8 puntos fijos como máximo. Esta restricción sobre el número de puntos fijos se justifica por el estudio anterior de las superfices que son a la vez $p$-hiperelípticas y $(q, n)$-gonales cíclicas.

Submitted by José María Montesinos Amilibia

Received: May 18, 2009. Accepted: November 11, 2009

Keywords: Automorphism groups of Riemann surfaces, hyperelliptic Riemann surfaces, $p$-hyperelliptic Riemann surfaces, $p$-gonal Riemann surfaces, Fuchsian groups

Mathematics Subject Classifications: $14 \mathrm{H}, 30 \mathrm{~F}$

(c) 2010 Real Academia de Ciencias, España 


\section{Introduction}

A compact Riemann surface $X$ of genus $g \geq 2$ is said to be p-hyperelliptic if $X$ admits a conformal involution $\rho$, called a $p$-hyperelliptic involution, such that $X / \rho$ is an orbifold of genus $p$. This notion has been introduced by H. Farkas and I. Kra in [16] where they also proved that for $g>4 p+1$, $p$-hyperelliptic involution is unique and so central in the group of all automorphisms of $X$. In [23] it has been proved that every two $p$-hyperelliptic involutions commute for $3 p+2 \leq g \leq 4 p+1$ and $X$ admits at most two such involutions if $g>3 p+1$.

In the particular cases $p=0,1, X$ are called hyperelliptic and elliptic-hyperelliptic Riemann surfaces respectively. Hyperelliptic Riemann surfaces and their automorphisms have received a good deal of attention in the literature. In [1] and [10] the authors determined the full groups of conformal automorphisms of such surfaces which made possible to classify symmetry types of such actions in [3]. The $p$-hyperelliptic $(p \geq 1)$ surfaces at large have been studied in [4-9, 13-15] and [24], where the most attention has been paid to a study of groups of automorphisms of such surfaces and their symmetries.

In [25], [21] and [22] the classification of conformal actions on $p$-hyperelliptic Riemann surfaces has been given, up to topological conjugacy, for $p=0,1$ and 2, respectively.

A closed Riemann surface $X$ which can be realized as a $n$-sheeted covering of the Riemann sphere is called $n$-gonal. Castelnuevo-Severi proved in [11] that if the genus $g$ of $X$ satisfies the inequality $g>(n-1)^{2}$ then a $n$-gonality automorphisms group is unique. In [19], Gromadzki justified that for $g \leq(n-1)^{2}, X$ has one conjugacy class of $n$-gonality automorphism groups in the group $\operatorname{Aut}(X)$ of automorphisms of $X$. This result has been proved using different techniques by González-Díez in [17]. The authors of [12] found the species of symmetries of real cyclic $p$-gonal Riemann surfaces while in [2], groups of automorphisms of cyclic trigonal Riemann surfaces have been determined.

A compact Riemann surface $X$ is called $(q, n)$-gonal if there exists a cyclic group of automorphism $C$ of $X$, called a $(q, n)$-gonal group of prime order $n$ such that $X / C$ has genus $q$. In [18], the conjugacy of $(q, n)$-gonal groups has been studied. Let us notice that the notion of $(q, 2)$-gonality coincides with $q$-hyperellipticity, whilst $(0, n)$-gonality coincides with $n$-gonality.

In this paper we study $p$-hyperelliptic Riemann surface $X$ which admits a conformal automorphism $\delta$, called $(q, n)$-gonal automorphism, of prime order $n>2$ such that $X / \delta$ has genus $q$ [18]. If the genus of $X$ is greater than $4 p+1$ then $\delta$ and $\rho$ commute. We give necessary and sufficient conditions on $p$ and $g$ for the existence of such a Riemann surface. We show that $\delta$ admits 3 or 4 fixed points if $q=0 ; 2-6$ if $q=1$ and at most 8 if $p<q$. We prove that if an automorphism group $G$ of a Riemann surface $X$ has a nontrivial centralizer then there exists a cyclic normal subgroup $H \subseteq G$ and we determine the presentation of a factor group $G / H$ in the case when a central automorphism of $X$ has at most 8 fixed points.

\section{Preliminaries}

A Fuchsian group $\Lambda$ is a discrete subgroup of the group of linear fractional transformations

$$
\mathrm{LF}(2, \mathbb{R})=\left\{z \mapsto \frac{a z+b}{c z+d}: a, b, c, d \in \mathbb{R}, a d-b c=1\right\},
$$

of the complex upper half-plane $\mathcal{H}$ onto itself with compact orbit space. This orbit space can be given an analytic structure such that the projection $\pi_{\Lambda}: \mathcal{H} \rightarrow \mathcal{H} / \Lambda$ is holomorphic. The algebraic structure of $\Lambda$ is determined by the signature $\sigma(\Lambda)=\left(g ; m_{1}, \ldots, m_{r}\right)$, where $g, m_{i}$ are integers verifying $g \geq 0, m_{i} \geq 2$. The signature determines the presentation of $\Lambda$ :

generators: $x_{1}, \ldots, x_{r}, a_{1}, b_{1}, \ldots, a_{g}, b_{g}$,

relations: $\quad x_{1}^{m_{1}}=\cdots=x_{r}^{m_{r}}=x_{1} \ldots x_{r}\left[a_{1}, b_{1}\right] \ldots\left[a_{g}, b_{g}\right]=1$.

Such set of generators is called a canonical set of generators and often, by abuse of language, its elements, canonical generators. Geometrically $x_{i}$ are elliptic elements which correspond to hyperbolic rotations and 
the remaining generators are hyperbolic translations. The integers $m_{1}, m_{2}, \ldots, m_{r}$ are called the periods of $\Lambda$ and $g$ is the genus of the orbit space $\mathcal{H} / \Lambda$. Fuchsian groups with signatures $(g ;-)$ are called surface groups and they are characterized among Fuchsian groups as these ones which are torsion free.

The group $\Lambda$ has associated to it a fundamental region $F_{\Lambda}$ whose area $\mu\left(F_{\Lambda}\right)=\mu(\Lambda)$, called the area of the group, is:

$$
\mu(\Lambda)=2 \pi\left(2 g-2+\sum_{i=1}^{r}\left(1-1 / m_{i}\right)\right) .
$$

If $\Gamma$ is a subgroup of finite index in $\Lambda$, then we have the Riemann-Hurwitzformula which says that

$$
[\Lambda: \Gamma]=\frac{\mu(\Gamma)}{\mu(\Lambda)} .
$$

By Riemann uniformization theorem, each compact Riemann surface $X$ of genus $g \geq 2$ can be represented as the orbit space of the hyperbolic plane $\mathcal{H}$ under the action of some Fuchsian surface group $\Gamma$. Furthermore, a group $G$ of automorphisms of a surface $X=\mathcal{H} / \Gamma$ can be represented as $G=\Lambda / \Gamma$ for another Fuchsian group $\Lambda$. The number of fixed points of an automorphism of $X$ can be calculated by the following theorem of Macbeath [20].

Theorem 1 Let $X=H / \Gamma$ be a Riemann surface with the automorphism group $G=\Lambda / \Gamma$ and let $x_{1}, \ldots, x_{r}$ be elliptic canonical generators of $\Lambda$ with periods $m_{1}, \ldots, m_{r}$ respectively. Let $\theta: \Lambda \rightarrow G$ be the canonical epimorphism and for $1 \neq g \in G$ let $\varepsilon_{i}(g)$ be 1 or 0 according as $g$ is or is not conjugate to a power of $\theta\left(x_{i}\right)$. Then the number $\mathrm{F}(g)$ of points of $X$ fixed by $g$ is given by the formula

$$
\mathrm{F}(g)=\left|\mathrm{N}_{G}(\langle g\rangle)\right| \sum_{i=1}^{r} \varepsilon_{i}(g) / m_{i},
$$

where $\mathrm{N}$ is a normalizer.

\section{3 -hyperelliptic Riemann surface with $(q, n)$-gonal automor- phism}

In this section we study Riemann surfaces of genera $g>1$ which are $p$-hyperelliptic and cyclic $(q, n)$-gonal simultaneously for a prime $n>2$ and a natural $q$. If $g>4 p+1$, then its $(q, n)$-gonal automorphism and $p$-hyperelliptic involution commute. The first theorem gives necessary and sufficient conditions on $p$ and $g$ for the existence of such a surface.

Theorem 2 There exists a p-hyperelliptic Riemann surface of genus $g \geq 2$ admitting ( $q, n)$-gonal automorphism commuting with a p-hyperelliptic involution if and only if $p=n \gamma+b(n-1) / 2$ and $g=$ $n q+a(n-1) / 2$ for some integers $\gamma, b$, a such that

$$
b=-2 \text { or } b \geq 0, \quad b \leq a \leq 2(b+1), \quad 0 \leq \gamma \leq(q+1) / 2 .
$$

Furthermore, the (q,n)-gonal automorphism admits $a+2$ fixed points.

Proof. Assume that a Riemann surface $X=\mathcal{H} / \Gamma$ admits $p$-hyperelliptic involution $\rho$ and $(q, n)$-gonal automorphism $\delta$. The groups $\langle\delta\rangle$ and $\langle\rho\rangle$ can be identified with $\Gamma_{\delta} / \Gamma$ and $\Gamma_{\rho} / \Gamma$, where $\Gamma_{\delta}$ and $\Gamma_{\rho}$ are Fuchsian groups containing $\Gamma$ as a normal subgroup of index $n$ and 2, respectively. By the RiemannHurwitz formula they have signatures

$$
\sigma\left(\Gamma_{\delta}\right)=(q ; n . r ., n) \quad \text { and } \quad \sigma\left(\Gamma_{\rho}\right)=(p ; 2, . . s ., 2) \text {, }
$$


where $s=2 g+2-4 p$ and $r=2+(2 g-2 n q) /(n-1)$. Thus $g=n q+a(n-1) / 2$ for $a=r-2$. If $\rho$ and $\delta$ commute then they generate the group $\mathbb{Z}_{2 n}$ which can be represented by $\Lambda / \Gamma$ for a Fuchsian group $\Lambda$ with the signature

$$
\left(\gamma ; 2, k_{1} ., 2, n, k_{2} ., n, 2 n, . k_{3} ., 2 n\right) .
$$

By the Riemann-Hurwith formula

$$
2 g-2=4 n \gamma-4 n+n k_{1}+2 k_{2}(n-1)+k_{3}(2 n-1)
$$

and according to Theorem 1

$$
n k_{1}=s-k_{3}, \quad 2 k_{2}=r-k_{3} .
$$

By substituting the last equalities to (4), we obtain $p=n \gamma+b(n-1) / 2$, for an integer $b$ such that $a=2 b+2-k_{3}$. Thus

$$
k_{1}=2 q+a-4 \gamma-2 b, \quad k_{2}=a-b, \quad k_{3}=2+2 b-a
$$

are nonnegative integers if and only if the inequalities (1) are satisfied.

Conversely, assume that $g=n q+a(n-1) / 2$ and $p=n \gamma+b(n-1) / 2$ for some integers $a, b$ and $\gamma$ satisfying the inequalities (1). Then there exists a Fuchsian group $\Lambda$ with the signature (3). Let $\theta: \Lambda \rightarrow\langle\rho\rangle \oplus\langle\delta\rangle$ be an epimorphism which maps all hyperbolic generators of $\Lambda$ onto $\rho \delta$, the first $k_{1}$ of elliptic generators onto $\rho$ and the remaining in the following way :

$$
\begin{gathered}
\underbrace{\delta \ldots \delta}_{\left(k_{2}+1\right) / 2} \underbrace{\delta^{-1} \ldots \delta^{-1}}_{\left(k_{2}-3\right) / 2} \delta^{-2} \underbrace{\rho \delta \ldots \rho \delta}_{\left(k_{3}+1\right) / 2} \underbrace{\rho \delta^{-1} \ldots \rho \delta^{-1}}_{\left(k_{3}-3\right) / 2} \rho \delta^{-2} \quad \text { if } k_{2} \equiv 1(2) \text { and } k_{3} \equiv 1(2), \\
\underbrace{\delta \ldots \delta}_{\left(k_{2}+1\right) / 2} \underbrace{\delta^{-1} \ldots \delta^{-1}}_{\left(k_{2}-3\right) / 2} \underbrace{-2} \underbrace{\rho \delta \ldots \rho \delta}_{k_{3} / 2} \underbrace{\rho \delta^{-1} \ldots \rho \delta^{-1}}_{k_{3} / 2} \quad \text { if } k_{2} \equiv 1(2) \text { and } k_{3} \equiv 0(2), \\
\underbrace{\delta \ldots \delta}_{k_{2} / 2} \underbrace{\delta^{-1} \ldots \delta^{-1}}_{k_{2} / 2} \underbrace{\rho \delta \ldots \rho \delta}_{\left(k_{3}+1\right) / 2} \underbrace{\rho \delta^{-1} \ldots \rho \delta^{-1}}_{\left(k_{3}-3\right) / 2} \rho \delta^{-2} \quad \text { if } k_{2} \equiv 0(2) \text { and } k_{3} \equiv 1(2), \\
\underbrace{\delta \ldots \delta}_{k_{2} / 2} \underbrace{\delta^{-1} \ldots \delta^{-1}}_{k_{2} / 2} \underbrace{\rho \delta \ldots \rho \delta}_{k_{3} / 2} \underbrace{\rho \delta^{-1} \ldots \rho \delta^{-1}}_{k_{3} / 2} \quad \text { if } k_{2} \equiv 0(2) \text { and } k_{3} \equiv 0(2) .
\end{gathered}
$$

Then the kernel of $\theta$ is a surface Fuchsian group $\Gamma$ of genus $g$ while $\theta^{-1}(\rho)$ and $\theta^{-1}(\delta)$ are Fuchsian groups with the signatures (2). Thus $\mathcal{H} / \Gamma$ is a $p$-hyperelliptic Riemann surface admitting $(q, n)$-gonal automorphism. It is easy to notice that for $k_{2}<3$ or $k_{3}<3$, such an epimorphism does not exist if and only if $k_{2}+k_{3}+\gamma=0$ or $k_{2}+k_{3}=1$. The first equality is never satisfied since if $k_{2}+k_{3}=0$ then $b=-2$ and $p=n(\gamma-1)+1$ what requires $\gamma \geq 1$. The second one occurs for $b=-1$ and therefore this value of $b$ is rejected.

Corollary 1 Let $X$ be a p-hyperelliptic Riemann surface of genus $g>4 p+1$. Then for any prime $n \geq 3$,

(i) $X$ can be realized as cyclic $n$-sheeted covering of the Riemann sphere if and only if $p=0$ and $g=n-1$ or $g=(n-1) / 2$ and its cyclic $n$-gonal automorphism admits 4 or 3 fixed points, respectively.

(ii) $X$ can be realized as cyclic $n$-sheeted covering of an elliptic curve if and only if $p=0$ and $g \in\{2 n-1,(3 n-1) / 2, n\}$ or $p=(n-1) / 2$ and $g \in\{3 n-2,(5 n-3) / 2\}$ and its $(1, n)$-gonal automorphism admits 4, 3, 2 or 6,5 fixed points, respectively.

Corollary 2 Let $X=\mathcal{H} / \Gamma$ be a Riemann surface of genus $g \geq 2$ which admits $p$-hyperelliptic involution $\rho$ and $(q, n)$-gonal automorphism $\delta$ for $p<n$. If $\delta$ and $\rho$ commute then $p=b(n-1) / 2, g=n q+a(n-1) / 2$ for integers $a, b$ in range $0 \leq b \leq 2$ and $b \leq a \leq 2 b+2$ and $a$ Fuchsian group $\Lambda$ such that $\langle\delta, \rho\rangle=\Lambda / \Gamma$ has a signature $\left(0 ; 2,{ }^{2 q+a-2 b}, 2, n, \stackrel{a-b}{.}, n, 2 n,{ }^{2 b+2-a}, 2 n\right)$. Furthermore, $\delta$ admits $a+2 \leq 8$ fixed points.

The last corollary is the inspiration for the next section in which we study the groups of automorphisms of a Riemann surface admitting a central automorphism with at most 8 fixed points. 


\section{Automorphism groups of a Riemann surface with nontrivial centralizer}

Let $G$ be an automorphism group of a Riemann surface $X$ of genus $g \geq 2$ admitting a central element $\delta$ of order $n$. If $z \in X$ is a fixed point of $\delta$, then $\delta$ preserves all points in the orbit $G z$. Assume that the stabilizer $\operatorname{Stab}(z)$ of $z$ is a cyclic group of order $m$ generated by $x \in G$. Then $n$ divides $m$ and $\langle\delta\rangle=\left\langle x^{m / n}\right\rangle$. Any element $g \in G$ permutes points of $G z$ and we shall assign a permutation $\sigma_{g} \in S_{k}$ to $g$, where $k=|G z|=|G| / m$. The permutation $\sigma_{x}$ splits into product of cycles of lengths $t_{1}, \ldots, t_{\beta}$, respectively, where $t_{j}$ divide $m$. Let $g_{1}, \ldots, g_{\beta}$ be different elements of $G$ for which $t_{j}$ are the smallest positive integers such that $x^{t_{j}} \in g_{j}\langle x\rangle g_{j}^{-1}$. Then

$$
G z=\left\{h_{1} z, \ldots, h_{\alpha} z, g_{1} z, x g_{1} z, \ldots, x^{t_{1}-1} g_{1} z, \ldots, g_{\beta} z, \ldots, x^{t_{\beta}-1} g_{\beta} z\right\},
$$

where $\alpha=k-\left(t_{1}+\cdots+t_{\beta}\right)$ and $h_{i} \in G$ normalize $\langle x\rangle$. We shall denote points $h_{i} z$ by $z_{i}$, in particular $z$ by $z_{1}$, and points $x^{l} g_{j} z$ by $z_{j, l}$. In order to determine the presentation of $G$ we shall need the following lemmata.

Lemma 1 Let $r_{i}$ be the smallest positive integer such that $g_{i}^{r_{i}} \in\langle x\rangle$ for $i=1, \ldots, \beta$. Then there exists an integer $b_{i}$ such that $b_{i} \equiv 1(n),\left(m / t_{i}, b_{i}\right)=1, b_{i}^{r_{i}} \equiv 1\left(m / t_{i}\right)$ and

$$
g_{i} x^{t_{i}} g_{i}^{-1}=x^{b_{i} t_{i}}
$$

Moreover, $g_{i}^{r_{i}}=x^{p_{i}}$ for some $p_{i}$ such that $p_{i} \equiv 0\left(t_{i}\right)$ and $b_{i} \equiv 1\left(m / \operatorname{gcd}\left(m, p_{i}\right)\right)$.

Proof. Assume that $x^{t_{i}}=g_{i} x^{t_{i} l_{i}} g_{i}^{-1}$ for an integer $l_{i}$ co-prime with $m / t_{i}$. Then there exist $a_{i}$ and $b_{i}$ such that $a_{i} m / t_{i}+b_{i} l_{i}=1$ and so $g_{i} x^{t_{i}} g_{i}^{-1}=x^{b_{i} t_{i}}$.

If $c$ is an integer such that $x^{c}$ and $g_{i}$ commute then $c \equiv 0\left(t_{i}\right)$ what implies $b_{i} \equiv 1(\mathrm{~m} / \operatorname{gcd}(m, c))$. Otherwise, a smaller power than $x^{t_{i}}$ would belong to $g_{i}\langle x\rangle g_{i}^{-1}$. In particular, $p_{i} \equiv 0\left(t_{i}\right), b_{i} \equiv 1\left(m / \operatorname{gcd}\left(m, p_{i}\right)\right)$ and $b_{i} \equiv 1(n)$. Finally, since $g_{i}^{r_{i}}$ and $x$ commute, it follows that $b_{i}^{r_{i}} \equiv 1\left(\mathrm{~m} / \mathrm{t}_{i}\right)$.

Lemma 2 For any $i$ in range $1 \leq i \leq \beta, g_{i}$ maps the set $F=\left\{z_{1}, \ldots, z_{\alpha}\right\}$ into $G z \backslash F$. Furthermore, if $g_{i}$ maps a point of $F$ into $z_{i^{\prime}, l}$ for some $1 \leq i^{\prime} \leq \beta$ and $1 \leq l \leq t_{i^{\prime}}$ then $t_{i}=t_{i^{\prime}}$ and $g_{i^{\prime}}$ maps a point of $F$ into $z_{i,-l}$.

PROOF. On a contrary, suppose that $g_{i}\left(z_{j}\right)=z_{j^{\prime}}$ for some $z_{j}, z_{j^{\prime}} \in F$. Then $z_{j}$ is a fixed point of $g_{i}^{-1} x g_{i}$. Thus $g_{i}^{-1} x g_{i} \in h_{j}\langle x\rangle h_{j}^{-1}=\langle x\rangle$ what implies $z_{i, 0}=z_{i, 1}$, a contradiction. So $g_{i}$ maps every $z_{j} \in F$ into some point $z_{i^{\prime} l} \in G z \backslash F$. Thus $x^{l} g_{i^{\prime}} x g_{i^{\prime}}^{-1} x^{-l}=g_{i} h_{j} x h_{j}^{-1} g_{i}^{-1} \in g_{i}\langle x\rangle g_{i}^{-1}$ what implies $t_{i}=t_{i^{\prime}}$.

Now let $g \in G$ be such an element that $g_{i^{\prime}}(g z)=z_{i,-l}$ Then $z_{i^{\prime}, 0}=g_{i^{\prime}} g\left(g^{-1} z\right)=x^{-l} g_{i} x^{s}\left(g^{-1} z\right)$ for some integer $s$ and so $z_{i^{\prime}, l}=g_{i}\left(x^{s} g^{-1} z\right)$ what implies $g^{-1} z=z_{j}$. Thus $g x g^{-1} \in\langle x\rangle$ what means that $g z \in F$.

By the proof of Lemma 2, we obtain the following

Corollary 3 If $\beta \neq 0$ then $\alpha \leq t_{1}+\cdots+t_{\beta}$ and $G$ is generated by $x$ and $g_{1}, \ldots, g_{\beta}$.

Lemma 3 If $g_{s}\left(z_{i_{0}, l_{0}}\right)=z$ for some $s, i_{0}$ and $l_{0}$ in range $1 \leq s, i_{0} \leq \beta$ and $1 \leq l_{0} \leq t_{i_{0}}$, respectively, then $t_{s}=t_{i_{0}}$. In particular, for $s=i_{0}$, the element $g=g_{i_{0}} x^{l_{0}-1}$ satisfies the relation $(g x)^{2}=1$ modulo $x^{t_{i}}$ and

$$
\begin{array}{ccrl}
g\left(z_{i, l}\right)=z_{i^{\prime}, l^{\prime}} & \text { if and only if } & g\left(z_{i^{\prime}, l^{\prime}+1}\right) & =z_{i, l-1}, \\
g\left(z_{j}\right)=z_{i, l} & \text { if and only if } & g\left(z_{i, l+1}\right)=z_{j}, \\
\text { if } g\left(z_{i, l+1}\right)=z_{i, l} & \text { then } t_{i} \text { is even and for } i=i_{0}, & x^{t_{i} / 2} g=g x^{1-l} g x^{l} .
\end{array}
$$


PROOF. Since $g_{s} x^{l_{0}} g_{i_{0}} \in\langle x\rangle$, it follows that $g_{s}^{-1} x g_{s}=x^{l_{0}} g_{i_{0}} x g_{i_{0}}^{-1} x^{-l_{0}}$ what implies $t_{i_{0}}=t_{s}$. If $s=i_{0}$ and $g=g_{i_{0}} x^{l_{0}-1}$ then $(g x)^{2} z=z$ and so $(g x)^{2}=x^{q}$ for some integer $q$. Thus $g^{2} x=g x^{q-1} g^{-1}$. On the other hand $x^{q}=g x g^{-1} g^{2} x$ implies that $g^{2} x=g x^{-1} g^{-1} x^{q}$. Consequently, $g x^{q} g^{-1}=x^{q}$ and so $q \equiv 0\left(t_{i_{0}}\right)$.

The statements (5) and (6) follow from the relation $(g x)^{2}=x^{q}$.

If $g\left(z_{i, l+1}\right)=z_{i, l}$ then $g x$ preserves point $z_{i, l}$ and so $g x=x^{l} g_{i} x^{r} g_{i}^{-1} x^{-l}$ for some $r$ not being a multiple of $t_{i}$. If $t_{i}$ is odd then rising the last equation to second power we obtain that $g x^{r^{\prime}} g^{-1} \in\langle x\rangle$ for some integer $r^{\prime}<t_{i}$ against our choice of $t_{i}$. For even $t_{i}, r=t_{i} / 2$ and additionally if $i=i_{0}$ then using the relation $(g x)^{2}=x^{q}$ we obtain $g x^{1-l} g x^{l}=x^{t / 2} g$.

Lemma 4 Let $i, j \in\{1, \ldots \beta\}, l \in\left\{1, \ldots, t_{j}\right\}$ and $l^{\prime} \in\left\{1, \ldots, t_{i}\right\}$.

(i) If $g \in G$ preserves point $z_{j, l}$ then $g^{t_{j}} \in\langle x\rangle$.

(ii) If $g_{i}\left(z_{j, l}\right)=z_{i, l^{\prime}}$, then $t_{j}$ divides $t_{i}$.

Proof. (i) By the assumption, $g \in x^{l} g_{j}\langle x\rangle g_{j}^{-1} x^{-l}$ and so $g^{t_{j}} \in\langle x\rangle$.

(ii) Here $g_{j}=x^{-l} g_{i}^{-1} x^{l^{\prime}} g_{i} x^{s}$ for some integer $s$. Thus $g_{j} x^{t_{i}} g_{j}^{-1} \in\langle x\rangle$ and so $t_{j}$ divides $t_{i}$.

Theorem 3 Let $G$ be a group of automorphisms of a Riemann surface $X$ admitting a central automorphism $\delta$ of order $n$ and suppose that $\delta$ admits $k \leq 8$ fixed points in the same orbit. Then for $k>1$, there exists an element $x \in G$ of order $m=|G| / k$ and an integer $t$ dividing $m$ such that $H=\left\langle x^{t}\right\rangle$ is a normal subgroup of $G, \delta \in H$ and $G / H$ has one of presentations listed in Table 1. For $k=1, G$ is a cyclic group.

Proof. Since $k<9$ then the sequence of parameters for the action of $G$ on such an orbit must be of the form $C_{k}=\left(\alpha ; t_{1}, t_{2}, t_{3}\right)$. First we show that some sequences are not possible. For, suppose that $t_{1} \neq t_{2}$ and $t_{1} \neq t_{3}$. Then by Lemma $3, g_{1}\left(z_{1, l_{0}}\right)=z$ for some $l_{0}=1, \ldots, t_{1}$ and we shall use $g=g_{1} x^{l_{0}-1}$ instead of $g_{1}$. Furthermore, according to Lemma 2, $g(F) \subset\left\{z_{1,0}, \ldots z_{1, t_{1}}\right\}$ and if $z_{1, l}$ is an image of a point from $F$ then $z_{1,-l}$ is also an image of a point from $F$. In particular, if $F$ contains only two points $z_{1}=z$ and $z_{2}$ then $x^{-l} g z=g\left(z_{2}\right)=x^{l} g z$ what requires $t$ even. Thus the sequences $C_{5}=(2 ; 3,0,0)$, $C_{7}=(2 ; 3,2,0)$ and $C_{7}=(2 ; 5,0,0)$ must be rejected. For $C_{8}=(3 ; 3,2,0)$, without lost of generality we can assume that $g\left(z_{2}\right)=x g z$ and $g\left(z_{3}\right)=x^{2} g z$. Thus by (6), $z_{2}=g\left(x^{2} g z\right)$ and $z_{3}=g(g z)$. So it remains that $g$ preserves or exchanges points $z_{2,0}$ and $z_{2,1}$ what by item (i) of Lemma 4 implies that $g^{2} \in\langle x\rangle$ or $g x=g_{2} x g_{2}^{-1}=x^{-1} g$, respectively. Thus not all points in $G z$ are different against the assumption. Similarly for $C_{8}=(3 ; 5,0,0)$, we can assume that $g\left(z_{2}\right)=x^{2} g z$ and $g\left(z_{3}\right)=x^{3} g z$. Thus by (6) and (7), $\sigma_{g}=(1,4,5)(2,6,8,3,7)$ and so $g^{3} \in\langle x\rangle$. However $g^{3}\left(z_{2}\right) \neq z_{2}$, a contradiction once again.

If $t_{i}$ does not divide $t_{1}$ for $i=1$ or 2 then by item (ii) of Lemma $4, g\left(z_{i, l}\right) \notin\left\{z_{1,1}, \ldots, z_{1, t_{1}}\right\}$. Thus for $C_{8}=(1 ; 5,2,0)$ and $C_{6}=(1 ; 3,2,0), g$ preserves points $z_{2,0}, z_{2,1}$ or exchanges them what has been shown is impossible. Using (7) for $C_{8}=(1 ; 3,2,2)$, we conclude that $\sigma_{g}$ is a product of cycles, one of which is $(1,2,3)$, and so $g^{3}=x^{p}$ for some integer $p$. However since $\sigma_{g}$ neither preserves nor exchanges points $z_{i, 0}$ and $z_{i, 1}$, it follows that $g^{3}\left(z_{2,0}\right) \neq z_{2, s}$ for $s=0,1$, a contradiction. The sequence $C_{8}=(1 ; 4,3,0)$ is also impossible since there does not exist $\sigma_{g}$ for which $g\left(z_{2, l}\right) \neq z_{1, l^{\prime}}$ and $g\left(z_{2, l+1}\right) \neq z_{2, l}$ for $l=0,1,2$ and $l^{\prime}=1, \ldots, 4$.

Since the case $(1 ; 3,2,2)$ is rejected and $k<9$, it follows that two parameters $t_{i}$ in the sequence $C_{k}=\left(\alpha ; t_{1}, t_{2}, t_{3}\right)$ can be equal if and only if $t_{i} \in\{0,2\}$ or $t_{i} \in\{0,3\}$ for $i=1,2,3$. We shall describe only the first possibility since the second one can be solved in the similar way. However in most cases all parameters $t_{1}, t_{2}$ and $t_{3}$ are different and first we concentrate on them. So assume that $t_{1}, t_{2}, t_{3}$ are different integers. Then by Lemma 3, there exist $i$ and $l$ in range $1 \leq i \leq 3$ and $1 \leq l \leq t_{i}$, respectively such that $g_{i}\left(z_{i, l}\right)=z$ and it is convenient to exchange $g_{i}$ for $g=g_{i} x^{l-1}$ which satisfies the relation $(g x)^{2} \equiv 1\left(x^{t}\right)$, for $t=t_{i}$. From now on we will write all relations modulo $x^{t}$ unless we say differently. Let us notice that $g\left(x g^{s} z\right)=x^{-1} g^{s-1} z$ for $s=1, \ldots, r$ and so $g(x g z)=z$. We find the permutation $\sigma_{g}$ and by 


\begin{tabular}{|c|l|l|}
\hline$k$ & Case & \multicolumn{1}{c|}{ Presentation of $\tilde{G}$} \\
\hline $2 \leq k \leq 8$ & $k .1$ & $\left\langle g: g^{k}=1\right\rangle$ \\
& $k .2$ & $\left\langle x, g: x^{2}=1, g^{k}=1,(g x)^{2}=1\right\rangle$ \\
4 & 4.3 & $\left\langle x, g: x^{3}=1, g^{3}=1,(g x)^{2}=1\right\rangle$ \\
5 & 5.3 & $\left\langle x, g: g^{4}=1, g x g^{-1}=x g x^{-1}, g^{2}=x^{2}(g x) x^{-2}\right\rangle$ \\
6 & 6.3 & $\left\langle x, g: x^{4}=1, g^{3}=1,(g x)^{2}=1\right\rangle$ \\
& 6.4 & $\left\langle x, g: x^{3}=1, g^{6}=1, x g^{3} x^{-1}=g x\right\rangle$ \\
7 & 6.5 & $\left\langle x, g: x^{2}=1, g^{3}=1,(g x)^{3}=1\right\rangle$ \\
8 & 7.3 & $\left\langle x, g: g^{3}=1, x^{3} g x^{-3}=g x^{2} g^{-1}, g x^{3} g^{-1}=x^{2}(g x) x^{-2}\right\rangle$ \\
& 8.3 & $\left\langle x, g: x^{3}=1, g^{4}=1,(g x)^{2}=1\right\rangle$ \\
& 8.4 & $\left\langle x, g: g^{6}=1, g x g^{-1}=x g^{2} x^{-1},(g x)^{2}=1\right\rangle$ \\
& 8.5 & $\left\langle x, g: x^{4}=1, g^{8}=1,(g x)^{2}=1,\left[g^{2}, x\right]=1\right\rangle$ \\
& 8.6 & $\left\langle x, g: g^{7}=1,(g x)^{2}=1, x^{2} g^{-1} x^{-2}=g x g^{-1}\right\rangle$ \\
& 8.7 & $\left\langle x, g: x^{3}=1, g^{4}=1,(g x)^{3}=1,\left[g^{2}, x\right]=1\right\rangle$ \\
& 8.8 & $\left\langle x, g: x^{3}=1, g^{4}=1,(g x)^{3}=g^{2},\left[g^{2}, x\right]=1\right\rangle$ \\
& 8.9 & $\left\langle x, g: x^{3}=1, g^{3}=1,(g x)^{2}=g^{-1} x g\right\rangle$ \\
& 8.10 & $\left\langle x, g: g^{3}=1,(g x)^{4}=1, x g x^{-1}=g x^{-1} g^{-1}\right\rangle$ \\
& 8.11 & $\left\langle x, g: x^{3}=1, g^{7}=1, g x=g^{-1} x g\right\rangle$ \\
& 8.13 & $\left\langle x, g: x^{2}=1, g^{4}=1,(g x)^{4}=1,\left[g^{2}, x\right]=1\right\rangle$ \\
& 8.14 & $\left\langle x, g: x^{2}=1, g^{8}=1,(g x)^{8}=1,\left[g^{2}, x\right]=1\right\rangle$ \\
& 8.15 & $\left\langle x, g: x^{4}=1, g^{4}=1,(g x)^{2}=1,\left[g^{2}, x^{2}\right]=1\right\rangle$ \\
& 8.16 & $\left\langle x, g 1, g_{2}: x^{2}=(g 1 x)^{2}=g_{1}^{4}=\left(g_{2} x\right)^{2}=1, g_{1}^{2}=g_{2}^{2}\right\rangle$ \\
\hline
\end{tabular}

Table 1. The presentation of the group $G / H$

consideration how it acts on points of $G z$ we obtain relations which determine the presentation of $G$. We consider the case with $t_{1}=t=4$ as an example, the remaining cases can be solved in the similar way. First we find the all possible values of $g^{2} z$. If $g^{2}=x g z$ then $g^{3} \in\langle x\rangle$ and by (5), $g\left(x^{2} g z\right)=x^{3} g z$. Using the relation $(g x)^{2}=1$ and $g^{3}=1$ we calculate that $\left(g x^{3} g\right) x\left(g x^{3} g\right)^{-1}=x^{-1}$ what means that $g\left(x^{3} g z\right)$ is a fixed point of $x$, say $z_{2}$. Thus by (6), $g\left(z_{2}\right)=x^{2} g z$. It is easy to notice that $G z$ cannot have any other points but $z, z_{2}, g z, \ldots, x^{3} g z$ since otherwise we get a contradiction with lemata. So we get the sequence $C_{6}=(2,4,0,0)$ for which $\sigma_{g}=(1,3,4)(2,5,6)$. By Lemma 1 and Corollary $3, G$ is generated by $x, g$ and admits a normal cyclic subgroup $H=\left\langle x^{4}\right\rangle$. By analyzing $\sigma_{g}$ we conclude that $\tilde{G}=G / H$ has the presentation 6.3 .

Next suppose that $g^{2}=x^{2} g z$. Then by (5), $x^{3} g z$ is a fixed point of $g$ and so by Lemma $4, g^{4} \in\langle x\rangle$. Thus $g\left(x^{2} g z\right)=g^{3} z=x g z$. Since $G z$ cannot have any additional points, it follows that $C_{5}=(1 ; 4,0,0)$, $\sigma_{g}=(1,2,4,3)$ and $\tilde{G}$ has the presentation 5.3 .

If $g^{2} z=x^{3} g z$ then $g x g^{2} z=g^{2} z$. Thus $g x$ preserves point $g^{2} z$ and so $g x=g^{2} x^{2} g^{-2}$. Consequently $z=g x g z=g^{2} x^{2} g^{-1} z=g^{2} x^{3} g z=g^{4} z$. So $g^{4} \in\langle x\rangle$ and we conclude that for $C_{5}=(1 ; 4,0,0)$, $\sigma_{g}=(1,2,5,3)$ and $\tilde{G}$ has the presentation $\tilde{G}=\left\langle x, g: x^{4}=1, g^{4}=1, g x g^{-1}=x^{2} g x^{-2}, g x^{2} g^{-1}=x g\right\rangle$ which is isomorphic to 5.3 .

If $g^{2} z=z_{2} \in F$ then $g\left(z_{2}\right)=x^{3} g z$. Thus according to Lemma 2, there exists $z_{3} \in F$ such that $x g z=g\left(z_{3}\right)$ and so by (6), $z_{3}=g\left(x^{2} g z\right)$. If $g\left(x^{3} g z\right)=x^{2} g z$ then by (7), $x^{2} g=g x^{3} g x^{2}$. However $x^{2} g(g z) \neq g x^{3} g x^{2}(g z)$ and so there exists one more point $z_{4} \in F$ such that $g\left(x^{3} g z\right)=z_{4}$. Thus $g\left(z_{4}\right)=$ 
$x^{2} g z$ and $\sigma_{g}=(1,5,2,8,4,7,3,6)$. So for $C_{8}=(4 ; 4,0,0), \tilde{G}$ has the presentation 8.5.

Finally suppose that $g^{2} z=z_{2,0}$. Then $g\left(z_{2,1}\right)=z_{1,3}$ and so by item (ii) of Lemma $4, t_{2}=2$. Let us consider all possible values of $g^{3} z$. If $g^{3} z=z_{1,1}$ then $g^{4} \in\langle x\rangle$ and $z_{2,1}=g\left(z_{1,2}\right)$. Furthermore, $g\left(z_{1,3}\right) \neq z_{1,2}$ since otherwise by (7), $x^{2} g=g x^{3} g x^{2}$. However by evaluation the last equality in $z_{1,0}$ we obtain different points. Thus there exists $z_{2} \in F$ such that $g\left(z_{1,3}\right)=z_{2}$ and consequently $g\left(z_{2}\right)=z_{1,2}$. So for $C_{8}=(2 ; 4,2,0), \sigma_{g}=(1,3,7,4)(2,5,8,6)$ and $\tilde{G}$ has the presentation 8.15.

If $g^{3} z=z_{1,2}$ then $z_{2,1}=g\left(z_{1,3}\right)$ and it remains that $g\left(z_{1,2}\right)=z_{1,1}$ or $g\left(z_{1,2}\right)=z_{2}$ for some $z_{2} \in F$. In the first case by (7), $x^{2} g=g^{2} x$ against the assumption that $z_{2,0}=g^{2} z$. The second one is also impossible since then $g\left(z_{2}\right)=z_{1,1}$. However there does not exist an integer $s$ such that $g^{2} x^{2} g\left(z_{2}\right)=x g x^{s}\left(z_{2}\right)$.

If $g^{3}=z_{2,1}$ then $g\left(z_{1,2}\right) \neq z_{1,1}$ and $g$ does not preserve $z_{1,2}$. Thus there exists $z_{2} \in F$ such that $g\left(z_{1,2}\right)=z_{2}$ what implies $g\left(z_{2}\right)=z_{1,1}$. So it remains that $g\left(z_{1,3}\right)=z_{1,2}$. However $x^{2} g\left(z_{2}\right) \neq$ $g x^{3} g x^{2}\left(z_{2}\right)$, a contradiction with (7).

Now we shall consider the sequences $C_{k}=\left(\alpha ; t_{1}, t_{2}, t_{3}\right)$, where $t_{i} \in\{0,2\}$ for $i=1,2,3$. First suppose that one of $g_{i}$, say $g_{1}$, satisfies $\left(g_{i} x\right)^{2} \in\langle x\rangle$. Then $x g_{1}^{s}=g_{1}^{-s} x$ for $s=1, \ldots, r$, where $r$ is the smallest positive integer such that $g_{1}^{r} \in\langle x\rangle$. Thus $g_{1}^{s} z$ is a fixed point of $x$ if and only if $r$ is even and $s=r / 2$, in this case we shall denote the point $g_{1}^{r / 2} z$ by $z_{2}$. In particular, if $r=k$ then $\alpha=1$ or 2 according to $k$ being odd or even, respectively, $g=g_{1}$ and $x$ generate $G$ and

$$
\tilde{G}=\left\langle x, g: x^{2}=1, g^{k}=1,(g x)^{2}=1\right\rangle .
$$

Since $g_{1}$ neither preserves nor exchanges points $z_{j, l}$ and $z_{j, l+1}$ for $j=1,2,3$ and $l=0,1$, it follows that we have the following possibilities for $r<k$ :

(i) $r=3, C_{6}=(2 ; 2,2,0), \sigma_{g_{1}}=(1,3,4)(2,5,6)$,

(ii) $r=4, C_{8}=(2 ; 2,2,2), \sigma_{g_{1}}=(1,3,2,4)(5,7)(6,8)$ or $(1,3,2,4)(5,6,7,8)$,

(iii) $r=4, C_{8}=(4 ; 2,2,0), \sigma_{g_{1}}=(1,5,2,6)(3,8,4,7)$. By analyzing $\sigma_{g_{1}}$ we conclude that $G$ is generated by $x$ and $g_{2}$. So we shall find $\sigma_{g_{2}}$ in order to determine the presentation of $G$. If $z=g_{2}\left(z_{1, l}\right)$ for some $l \in\{0,1\}$ then not all points in $G z$ are different. So we can assume that $\left(g_{2} z\right)^{2} \in\langle x\rangle$.

(i) Since $x g_{1}$ preserves point $z_{2,0}$, it follows that $x g_{1}=g_{2} x g_{2}^{-1}$. Thus $g_{1}=g_{2}^{-2}$ and so $g_{2}^{6} \in\langle x\rangle$. Consequently $\tilde{G}$ has the presentation (8), where $k=6$ and $g=g_{2}$.

(ii) Let us notice that the first permutation leads to a contradiction. Indeed, since $g_{1}^{2}$ preserves $z_{2,0}$, it follows that $g_{1}^{2}=g_{2} x g_{2}^{-1}$. Thus if $z^{\prime}$ is a fixed point of $g_{1}^{2}$ then $g_{2}^{-1}\left(z^{\prime}\right) \in F$. However $g_{1}^{2}$ admits 4 fixed points and therefore not all points in $G z$ are different. By the second permutation, $x g_{1}$ preserves $\left(z_{2,0}\right)$, what implies $g_{1}=x g_{2} x g_{2}^{-1}=g_{2}^{-2}$. Thus $g_{2}^{8} \in\langle x\rangle$ and so $\tilde{G}$ has the presentation (8), where $k=8$ and $g=g_{2}$.

(iii) Since $x g_{1}^{2}$ preserves $z_{2,0}$, it follows that $x g_{1}^{2}=g_{2} x g_{2}^{-1}$ and so $g_{1}^{2}=g_{2}^{2}$. Thus we conclude that $\sigma_{g_{2}}=(1,7,2,8)(3,5,4,6)$ and $\tilde{G}$ has the presentation 8.16.

Next suppose that $\left(g_{i} x\right)^{2} \notin\langle x\rangle$ for $i=1,2,3$. Then without lost of generality we can assume that $z_{2, l}=x^{l} g^{-1} z$ for $l=0,1$ and $g=g_{1}$. Let us notice that $g\left(z_{2,1}\right) \neq z_{1,1}$ since otherwise $g x g^{-1}=x g x^{s}$ for some integer $s$ and evaluation the last equality in $z_{1,0}$ implies that $g\left(z_{1, s}\right)=z_{1,1}$, a contradiction. Since $g$ does not preserve any points $z_{i, l}$ and $g\left(z_{2, l}\right) \neq z_{2, l+1}$ for $i=1,2,3$, it follows that the sequences $C_{5}=(1 ; 2,2,0)$ and $C=(3 ; 2,2,0)$ are impossible. For $C_{6}=(2 ; 2,2,0), g\left(z_{2,1}\right)=z_{2}$ and $g\left(z_{2}\right)$ is one of points $z_{1,1}, z_{2,0}, z_{2,1}$. Using Lemma 2 we check that all possibilities provide a contradiction except the first one. Here $\sigma_{g}=(1,3,5)(2,4,6)$ and we conclude that $\tilde{G}$ has the presentation 6.5. For $C_{8}=(2 ; 2,2,2)$ we obtain the presentation 8.12. Finally for $C_{8}=(4 ; 2,2,0)$, since $g(F)=G z \backslash F$, we can assume that $z_{2}=g^{2} z$ and so $\left[g^{2}, x\right]=1$. Furthermore, $g^{3} z \in\left\{z_{1,1}, z_{2,0}, z_{2,1}\right\}$. If $g^{3} z=z_{1,1}$ then $z_{1,0}=x g^{3} z=g^{2} x g z$ what implies $z_{1,1}=z_{2,0}$, a contradiction. If $g^{3} z=z_{2,0}$ then $g^{4} \in\langle x\rangle$ and so $g^{2}(x g z)=x g^{3} z$ and $g^{2}\left(x g^{3} z\right)=x g z$. Thus $\sigma_{g}=(1,5,2,7)(3,8,4,6)$ and $\tilde{G}$ has the presentation 8.13. If $g^{3} z=z_{2,1}$ then $g^{-1} z=x g^{3} z=g(g x g z)$. Here $\sigma_{g}=(1,5,2,8,4,6,3,7)$ and $\tilde{G}$ has the presentation 8.14 . 
If $\beta=0$, then $G$ is generated by two elements $g$ and $x,\langle x\rangle$ is a normal subgroup of $G$ and $\tilde{G}=$ $\left\langle g: g^{k}=1\right\rangle$.

By corollaries 1 and 3 we obtain the following

Corollary 4 Let $X$ be a p-hyperelliptic Riemann surface with a central $(q, n)$-gonal automorphism $\delta$. Then for $p<n$ or $q=0,1, \delta$ has at most 8 fixed points and an automorphism group of $X$ is determined by Theorem 3.

Acknowledgement. The author supported by the Research Grant N. N201 366436 of the Polish Ministry of Sciences and Higher Education.

\section{References}

[1] BRandt, R. And Stichtenoth, H., (1986). Die Automorphismengruppen hyperelliptischer Kurven, Manuscripta Math., 55 ,1, 83-92. DOI: 10.1007/BF01168614

[2] Bujalance, E.; Cirre, F. J. and Gromadzki, G., (2009). Groups of automorphisms of cyclic trigonal Riemann surfaces, J. Algebra, 322, 4, 1086-1103. DOI: 10.1016/j.jalgebra.2009.05.017

[3] Bujalance, E.; Cirre, F. J.; Gamboa, J. M. And Gromadzki, G., (2001). Symmetry types of hyperelliptic Riemann surfaces, Mém. Soc. Math. Fr. (N.S.), 86.

[4] Bujalance, E. And Costa, A. F., (1997). On symmetries of $p$-hyperelliptic Riemann surfaces, Math. Ann., 308, 1, 31-45. DOI: 10.1007/s002080050062

[5] Bujalance, E. And Etayo, J. J., (1988). Large automorphism groups of hyperelliptic Klein surfaces, Proc. Amer. Math. Soc., 103, 3, 679-686. DOI: 10.2307/2046834

[6] Bujalance, E. AND Etayo, J. J., (1988). A characterization of $q$-hyperelliptic compact planar Klein surfaces, Abh. Math. Sem. Univ. Hamburg, 58, 1, 95-102. DOI: 10.1007/BF02941371

[7] Bujalance, E.; Etayo, J. J. And Gamboa, J. M., (1986). Surfaces elliptiques-hyperelliptiques avec beaucoup d'automorphismes, C. R. Acad. Sci. Paris Sér. I Math., 302, 10, 391-394

[8] Bujalance, E.; Etayo, J. J. And Gamboa, J. M., (1987). Topological types of $p$-hyperelliptic real algebraic curves. Math. Z., 194, 2, 275-283.

[9] Bujalance, E., Etayo, J. J., Gamboa, J. M. and GromadzKi, G., (1990). Automorphisms Groups of Compact Bordered Klein Surfaces. A Combinatorial Approach, Lecture Notes in Math., 1439, Springer Verlag.

[10] Bujalance, E.; Gamboa, J. M. And GromadzKi, G., (1993). The full automorphisms group of hyperelliptic Riemann surfaces, Manuscripta Math, 79, 1, 267-282. DOI: 10.1007/BF02568345

[11] Castelnuevo, G., (1906). Sulle serie algebriche di gruppi di punti apparteneti ad una curve algebraica, Rendiconti della R. Academia dei Lincei, Series 5, XV, (memorie scelte p. 509).

[12] Costa, Antonio F. And Izquierdo, Milagros, (2004). Symmetries of real cyclic p-gonal Riemann surfaces, Pacific J. Math., 213, 2, 231-243. DOI: 10.2140/pjm.2004.213.231

[13] Estrada, B., (2000). Automorphism groups of orientable elliptic-hyperelliptic Klein surfaces, Ann. Acad. Sci. Fenn. Math., 25 , 439-456.

[14] EstradA, B., (2002). Geometrical characterization of p-hyperelliptic planar Klein surfaces, Comput. Methods Funct. Theory, 2, 1, 267-279.

[15] Estrada, B. And Martínez, E., (2001). On $q$-hyperelliptic k-bordered tori, Glasg. Math. J., 43, 3, 343-357. DOI: $10.1017 /$ S0017089501030142 
[16] Farkas, H. M. And Kra, I., (1980). Riemann Surfaces, Graduate Text in Mathematics, Springer-Verlag.

[17] GonzÁlez-Díez, G., (1995). On prime Galois coverings of Riemann sphere, Ann. Mat. Pura Appl., 168, IV, 1-15. DOI: 10.1007/BF01759251

[18] Gromadzki, G.; Weaver, A. and Wootton, A., On gonality of Riemann surfaces, Geom. Dedicata, to appear. DOI: 10.1007/s10711-010-9459-X

[19] Gromadzki, G., (2008). On conjugacy of p-gonality automorphisms of Riemann surfaces, Rev. Mat. Complut., 21, 1, 83-87.

[20] Macbeath, A. M., (1973). Action of automorphisms of a compact Riemann surface on the first homology group, Bull. London Math. Soc., 5, 1, 103-108. DOI: 10.1112/blms/5.1.103

[21] Tyszkowska, E., (2005). Topological classification of conformal actions on elliptic-hyperelliptic Riemann surfaces, J. Algebra, 288, 2, 345-363. DOI: 10.1016/j.jalgebra.2005.03.024

[22] TyszKowska, E., (2008). Topological classification of conformal actions on 2-hyperelliptic Riemann surfaces, Bull. Inst. Math. Acad. Sinica, 33, 4,345-368.

[23] Tyszkowska, E., (2005). On p-hyperelliptic involutions of Riemann surfaces, Beiträge zur Algebra und Geometrie Contributions to Algebra and Geometry, 46, 2, 581-586.

[24] Tyszkowska, E. And Weaver, A., (2008). Exceptional points in the eliptic-hyperelliptic locus, J. Pure Appl. Algebra, 212, 1415-1426.

[25] Weaver, A., (2004). Hyperelliptic surfaces and their moduli, Geom. Dedicata, 103, 69-87.

\author{
Ewa Tyszkowska \\ Institute of Mathematics, \\ Gdańsk University, \\ Wita Stwosza 57, \\ 80-952 Gdańsk, \\ Poland
}

